\title{
COURSE AND PROGNOSIS OF SARCOIDOSIS IN A REFERRAL SETTING IN TURKEY; ANALYSIS OF 166 PATIENTS
}

\author{
Sedat Uygun, Halil Yanardag, Yesari Karter, Sabriye Demirci
}

Istanbul University, Cerrahpaşa Medical Faculty, Istanbul, Turkey: Department of Internal Medicine

\begin{abstract}
Summary: Backround: Clinical manifestations and prognosis of sarcoidosis are heterogenous and the prevalence varies depending on the country, area and race evaluated. Also the organs involved and courses of the disease differ greatly between countries, areas, races and individuals. Aims: To investigate the sociodemographic characteristics, clinical presentation and symptoms and to determine the severity and prognosis of sarcoidosis in Turkey as a referral center. Methods: Between January and July 2003 we retrospectively evaluated the outcome of the patients with sarcoidosis whose first clinical visits were between 1965 and 2003 in the multidisciplinary referral setting RS at Cerrahpasa Medical Faculty of the University of Istanbul. Data collected about each patient included sociodemographic characteristics, clinical presentation, symptoms, date of diagnosis, date and age of onset, method and stage of disease at the date of diagnosis and at the date of last evaluation; the mortality and survival rate were calculated. One hundred and sixty six consecutive patients whose first clinical visits were between 1965 and 2003 in the multidisciplinary RS at Cerrahpasa Medical Faculty were enrolled. We contacted every patient in our cohort by telephone calls or home visits. All those contacted were called back to outpatient clinic for a formal evaluation between June and September 2003. A formal physical examination and thorax radiography were performed in patients who came to the hospital. Their radiological stage, signs, symptoms and associated extrapulmonary manifestations were recorded. Results: At the initial presentation, the mean age of diagnosis was 40.3 years. The 31-40 age group is the group with the highest number of patients. Coughing was the most frequent symptom and erythema nodosum was the most frequent sign in both sexes. Thirty eight percent of patients had extrathoracic involvement. The most frequent extrapulmonary site of involvement was skin. The mortality rate was $11.6 \%(10.8 \%$ in females and $13 \%$ in males). Comorbidity was 3\% (5 females, 1 male). Females, youngs and patients without extrathoracic involvement had higher survival rates. Conclusion: Clinical characteristics, course and prognosis of sarcoidosis vary in different studies. The results may vary accordingly to ethnic, geographic, social and economic conditions.
\end{abstract}

Key words: Sarcoidosis, Prognosis, Referral centre

\section{Introduction}

Sarcoidosis is a multisystem disease of unknown etiology characterised by formation of non-caseating granulomas in the affected organs. It may involve the lung, skin, eye, myocardium, osteoarticular, reticuloendothelial and central nervous system $(8,24)$. Many possible etiologic factors, including infectious, genetic, autoimmune and environmental agents have been proposed. The incidence rate for sarcoidosis ranges from 1.4 per 100000 in Japan to 9.6 per 100000 in Germany. The prevalence rates of sarcoidosis ranges from 0.04 per 100000 in Spain to 64 per 100000 in Sweden. Blacks in USA have a prevalence of 64 per 100 000 . The mortality rates of sarcoidosis have been reported to vary from 2.2 to $28.1 \%$ (11). A range of 1 to $5 \%$ was provided in a recently published joint statement of the Ame- rican Thoracic Society, European Respiratory Society and World Association of Sarcoidosis and Other Granulomatous Diseases (2). The authors of the joint statement ascribed the five fold range of sarcoidosis mortality to patient source (referral bias) and genetic factors principally ethnicity: the individuals of Scandinavian descent are believed the experience the most, and the African Americans the least favorable course (24). The population served by referral settings RS are a composite of unselected patients derived from their serviced community plus a variable proportion of patients who have been referred, most often because of the expectation or realization of an unfavorable course. Most reports on the course and prognosis of sarcoidosis originate in speciality clinics, university settings and tertiary care facilities in which high mortality is likely to mirror a referral bias (36). 
In our current study, we evaluated the clinical and laboratory characteristics and mortality and morbidity in 166 patients with sarcoidosis who have been followed in our referral setting since 1965 . The major goal was to investigate the sociodemographic characteristics, clinical presentation and symptoms and to determine the severity and prognosis of sarcoidosis in our country as a RS.

\section{Materials and methods}

Between January and July 2003 we respectively evaluated the outcome of 166 consecutive patients whose first clinical visits were between 1965 and 2003 in the multidisciplinary referral setting at Cerrahpasa Medical Faculty of the University of Istanbul. This dedicated clinic 'Department of Lung Diseases' was founded in 1965 and had been the first and only RS of its kind in Turkey. It has been acting to diagnose or to confirm the diagnosis firstly, to search for the extrapulmonary manifestations and to treat and follow up these patients. The referrals to our center were either from the various departments of our faculty or from outside physicians. All patients are initially evaluated by an internist. The records were reviewed initially to confirm the diagnosis of sarcoidosis. The medical records and when indicated, the radiographic findings of each person with a diagnosis of sarcoidosis were reviewed by the senior author. Patients who were suspected of having sarcoidosis underwent a biopsy of the most accessible involved organ. Extrapulmonary sarcoidosis, for example hepatic, was confirmed by a compatible clinical picture supported by biopsy of the affected organ showing noncaseating granuloma and absence of concurrent or subsequent evidence of an identifiable cause $(14,23)$.

The patients are referred to the departments of neurology, dermatology or others when needed and all management problems are discussed. All the processes are free in our center.

We attempted to contact every patient in our cohort by telephone calls or by home visits. All those contacted were called back to outpatient clinic for a formal evaluation between June and September 2003. A formal physical examination and thorax radiography were performed. Their radiological stage, signs, symptoms and associated extrapulmonary manifestations were recorded.

We were planning to enroll all recorded patients (166 patients; 107 females, 59 males), but we could not contact with 28 patients. One hundred sixty six patients were enrolled to analyse the sociodemographic characteristics, clinical presentation, symptoms, date of diagnosis, date and age of onset, method and stage of disease at the date of diagnosis, but mortality and survival analysis were calculated among 138 patients whose outcome informations had been available. Sixteen of the cases were dead (11.6\%). Ten were females $(10.8 \%)$ and 6 were males $(13 \%)$. We could contact with 122 patients ( 82 females, 40 males) and take them to our outpatient department (Table 1).

The data collected about each patient included sociodemographic characteristics, clinical presentation, symptoms, date of diagnosis, date and age of onset, method and stage of disease at the date of diagnosis and at the date of last evaluation. The date of diagnosis is the date of onset or the date of the first abnormal finding. The date of onset was taken as the time symptoms and signs developed that were latter attributed to sarcoidosis or, in an asymptomatic person, the date of the first abnormal finding, usually on chest radiography. The date of last evaluation is the date of last clinical evaluation of the signs and symptoms of sarcoidosis and/or the date of last chest radiographic procedure. The mean follow up period was 10.7 years, (range 1-38).

The 122 contacted patients were taken to our outpatient clinic between June and September 2003. Chest radiographs were obtained. Radiographic staging followed the guidelines of Siltzbach et al (36). Their radiological stage, evaluation of these stages, signs, symptoms and associated extrapulmonary manifestations were recorded.

Organ involvement was determined in each patient using an assessment system based on findings from history, physical examination, chest x-ray and laboratory testing including blood counts, liver function tests and serum calcium.

The biopsy data and laboratory results were compared with the data for organ involvement in the suspected cases. If there was a discrepancy, the individual case was reviewed to exclude any other pathology.

The frequency of the symptoms, mortality, morbidity and prognosis were investigated and comparisons were made between sex and age groups.

Quantitative variables were expressed as mean +/- SD. Differences between the Kaplan-Meier curves were calculated by the long rank tests. Chi square tests were used to analyse nonparametric data.

\section{Results}

One hundred thirty eight patients (92 female and 46 male) were analysed in our current evaluation. The mean

Tab. 1: Distribution of the patients according to gender.

\begin{tabular}{|l|c|c|c|c|c|c|c|c|}
\hline & $\begin{array}{c}\text { Initial } \\
(\mathrm{n})\end{array}$ & $\begin{array}{c}\text { Initial } \\
(\%)\end{array}$ & $\begin{array}{c}\text { Lost } \\
(\mathrm{n})\end{array}$ & $\begin{array}{c}\text { Lost } \\
(\%)\end{array}$ & $\begin{array}{c}\text { Death } \\
(\mathrm{n})\end{array}$ & $\begin{array}{c}\text { Death } \\
(\%)\end{array}$ & $\begin{array}{c}\text { Current } \\
(\mathrm{n})\end{array}$ & $\begin{array}{c}\text { Current } \\
(\%)\end{array}$ \\
\hline Male & 59 & 36 & 13 & 46 & 6 & 38 & 40 & 33 \\
\hline Female & 107 & 64 & 15 & 54 & 10 & 62 & 82 & 67 \\
\hline Total & 166 & 100 & 28 & 100 & 16 & 100 & 122 & 100 \\
\hline
\end{tabular}


age was 51.4 yrs in males and 50.2 yrs in females with a range from 29 to 82 in males and 26 to 84 in females. All the patients were Caucasians.

At the initial presentation (166 patients; 107 females, 59 males) the mean age of diagnosis were $40.3 \mathrm{yrs}$ (40.8 in

Tab. 2: The distribution of the patients according to age groups.

\begin{tabular}{|c|c|c|c|}
\hline $\begin{array}{c}\text { Age Groups } \\
(\mathrm{yrs})\end{array}$ & $\begin{array}{c}\text { Males } \\
(\mathrm{n})\end{array}$ & $\begin{array}{c}\text { Females } \\
(\mathrm{n})\end{array}$ & $\begin{array}{c}\text { Total } \\
(\mathrm{n})\end{array}$ \\
\hline $10-20$ & - & - & - \\
\hline $21-30$ & 2 & 4 & 6 \\
\hline $31-40$ & 13 & 20 & 33 \\
\hline $41-50$ & 11 & 21 & 32 \\
\hline $51-60$ & 9 & 17 & 26 \\
\hline $61-70$ & 7 & 20 & 27 \\
\hline $71-80$ & 2 & 8 & 10 \\
\hline $81-90$ & 2 & 2 & 4 \\
\hline Total number & 46 & 92 & 138 \\
\hline
\end{tabular}

Tab. 3: The mortality rate of age groups.

\begin{tabular}{|c|c|c|c|}
\hline $\begin{array}{c}\text { Age Groups } \\
(\mathrm{yrs})\end{array}$ & $\begin{array}{c}\text { Males } \\
(\mathrm{n})\end{array}$ & $\begin{array}{c}\text { Females } \\
(\mathrm{n})\end{array}$ & $\begin{array}{c}\text { Total } \\
(\mathrm{n})\end{array}$ \\
\hline $10-20$ & - & - & - \\
\hline $21-30$ & - & - & - \\
\hline $31-40$ & 2 & 1 & 3 \\
\hline $41-50$ & - & - & - \\
\hline $51-60$ & - & 3 & 3 \\
\hline $61-70$ & 3 & 5 & 8 \\
\hline $71-80$ & - & - & - \\
\hline $81-90$ & 1 & 1 & 2 \\
\hline Total number & 6 & 10 & 16 \\
\hline
\end{tabular}

Tab. 4: The stages of the patients at the date of diagnosis.

\begin{tabular}{|c|c|c|}
\hline STAGE & N & $\%$ \\
\hline I & 84 & 50 \\
\hline II & 64 & 39 \\
\hline III & 15 & 9 \\
\hline IV & 3 & 2 \\
\hline Total & 166 & 100 \\
\hline
\end{tabular}

Tab. 5: Prognosis of patients by stage. females and 39.3 in males; $p>0.05)$. Eighty five ( $51 \% ; 53$ female, 35 male) were younger than 40 while 78 were older (47\%; 54 females, 24 males). Three of the women were 40 years old. The 31-40 yrs age group including 33 patients ( $24 \%$; 20 females and 13 males) is the group with the highest number of patients and the 41-50 yrs group follows it with 32 patients (23\%; 21 females, 11 males) (Table 2).

At the initial clinical presentation to our center 19 of the 166 patients (11\%) had no symptom or sign. Coughing was the most frequent symptom (71\% in females and $34 \%$ in males) and erythema nodosum was the most frequent sign ( $77 \%$ in females and $40 \%$ in males) in both sexes.

At the initial diagnosis 103 patients (62\%) (62\% female, $38 \%$ male had intrathoracic sarcoidosis while 66 (38\%) (65\% female, 35\% male) had extrathoracic involvement. Sixty of the patients ( $91 \%$ ) (65\% female, $35 \%$ male) with extrapulmonary involvement had also intrathoracic involvement. The most frequent extrapulmonary involvement was the skin (20.6\% in females and $16.9 \%$ in males). It was followed by peripheral lymphadenopathy, heart, liver, brain and eye in order. There was no significant difference between the males and females as extrapulmonary involvement is considered.

The mortality rate in 138 patients with the diagnosis of sarcoidosis followed between 1965 and 2003 was $11.6 \%$ ( $10.8 \%$ in females and $13 \%$ in males). The mean number of years of follow up 10.7 years (11.3 in females, 9.5 in males; $\mathrm{p}>0.05$ ). Sixteen of our patients ( 6 males, 10 females $)$ died during the follow up period of 38 years. Seven of them (44\%) due to sudden cardiac death (possibly heart involvement), $4(25 \%)$ due to respiratory failure, and 5 due to causes other than sarcoidosis. Three of the sudden cardiac deaths $(42 \%)$ were female and $4(58 \%)$ were male. Two of the patients with respiratory failure were females $(50 \%)$. The 61-70 yrs age group included the highest number of deaths (50\%) (3 males, 5 females). Mortality rates of age groups is given in table 3 .

Comorbidity was found in 5 of the 138 patients (3\%) (4 females, 1 male). The associated diseases in females were breast carcinoma, hepatocellular carcinoma, hepatocellular carcinoma with lung metastasis, adenocarcinoma without a primary focus and a gynecological malignity. The male with the associated comorbidity had lung carcinoma and CREST syndrome.

\begin{tabular}{|l|c|c|c|c|c|}
\hline Authors & $\begin{array}{c}\text { Follow-up } \\
\text { period (yrs) }\end{array}$ & $\begin{array}{c}\text { Resolved } \\
\text { Stage I (\%) }\end{array}$ & $\begin{array}{c}\text { Resolved } \\
\text { Stage II-III (\%) }\end{array}$ & $\begin{array}{c}\text { Mortality } \\
\%\end{array}$ & Source of cases \\
\hline Jerome $^{1}$ & $1-30$ & 66 & 53 & 0 & nRS \\
\hline Romer $^{8}$ & $1-10$ & 57 & 44 & 0 & nRS \\
\hline Smellie,Hoyle $^{28}$ & $2-22$ & 61 & 51 & 6 & RS \\
\hline Hannuksela et al $^{40}$ & $3-9$ & 78 & 41 & 4 & RS \\
\hline Scadding $^{27}$ & $5($ mean) & 82 & 51 & 2.2 & RS \\
\hline James $^{11}$ & $2.5-20$ & 65 & 41 & 13 & RS \\
\hline Present study & $1-38$ & 54 & 53 & & RS \\
\hline
\end{tabular}

RS: Referral setting nRS: nonreferral setting 
The survival rate of females was significantly higher than the males $(32.7$ yrs versus $21.3 \mathrm{yrs} ; \mathrm{p}<0.05)$. The patients who were younger than $40 \mathrm{yrs}$ and the patients without extrapulmonary involvement had better survival rates. (34.7 yrs versus 21.2 yrs ; $p<0.005$ and 33.6 yrs versus $26.1 \mathrm{yrs} ; \mathrm{p}<0.05$ respectively). There was no relation between the survival rate and the radiological staging and comorbidity.The stages on the date of diagnosis which were set according to the radiological scanning are given in table 4. It is seen that the proportion of the stages was $50 \%, 39 \%$, $9 \%$ and $2 \%$ in stages I, II, III and IV respectively.

In our current evaluation $54,2 \%$ of the patients in stage I resolved and $45.8 \%$ progressed to stage II. In stage II 52\% resolved and $20 \%$ progressed to stage III; $28 \%$ remained in the same stage. In stage III 55\% resolved and $45 \%$ progressed to stage IV (Table 5).

\section{Discussion}

Sarcoidosis has a variable clinical presentation and course. Most of the patients are diagnosed incidentally at the centers where x-rays are taken due to other complaints and no other sign or symptom is seen. Only less than half of the cases can be diagnosed premortem. Some of the cases show remission in a short time, but the disease may relapse, while some of them represent a complete remission after a long time of disease. Five to ten percent of the patients show progression to fatal pulmonary fibrosis $(11,14$, 25,32,34,36).

Prior studies emphasize the disease onset between the ages of 20 and 40 yrs $(6,22,39)$. The peak age group in the ACCES study was 35 to 45 yrs and approximetaly $1 / 3$ of the recruited patients were 50 yrs or older. Many patients above 40 yrs were included in some other studies from Europe, India and Japan $(5,9,12,15,21,27)$. Some of the studies have commented on a bimodal age occurence with the second peak seen in patients over 50 yrs old especially in women $(14,15,21,28 \mathrm{et}$ al.). Hillerdal observed an initial peak of disease at about age $30 \mathrm{yr}$ and a second peak in incident cases at about age $50 \mathrm{yrs}$, which was more prominent in women (15). Baughman et al studied the clinical characteristics of 736 patients with sarcoidosis within 6 months of diagnosis from 10 clinical centers in USA. Sixty four $\%$ of the group were female and $46 \%$ were less than 40 yrs old. The average women age at the entry to the study (when diagnosed) was 40 yrs or older whereas men tended to be below 40 yrs (3). In our study the mean age at the date of diagnosis was 40.3 yrs and there was no significant difference between the males and females ( 40.8 in females and 39.3 in males; $\mathrm{p}>0.05$ ). Fifty-one percent (30\% female, $21 \%$ male) were under than 40 while $47 \%$ were older $(33 \%$ females, $14 \%$ males). Three of the women were 40 years old (they are not included in the above age groups). Our results are similar to Baughman's. The 31-40 age group of Baughman including 30 patients ( 19 females and 11 males) is the group with the highest number of patients (similar with ACCES study) (3).

In our study the $31-40$ age group including 33 patients (24\%) (20 females and 13 males) is the group with the highest number of patients and the 41-50 group follows it with 32 patients (23\%) (21 females, 11 males).

Prior studies have suggested that the clinical manifestations of sarcoidosis differ in different parts of the world (20,36). Sarcoidosis in Japan is reported to have a much higher likelihood of ocular and cardiac diseases than in the West (1). Some authors suggested that these differences have environmental origin, but most authors consider them to be due to genetic predispositions. Certain human leukocyte antigens are associated with different presentations of the disease and prognosis $(4,28)$.

Jerome and Johnson evaluated the course and prognosis of 86 patients with sarcoidosis over a 10-year period of observation in a nonreferral setting in Portland and reported that respiratory symptoms were more frequent than any other $(29.1 \%)$. When compared with our results, despite the different rates the most frequent symptoms are similar (23).

Baughman et al (3) reported that the type and frequency of organ involvements were changing according to race, sex and age (eye in blacks, abnormal calcium metabolism in men, neurologic involvement in women). Those under $40 \mathrm{yrs}$ of age at the time of diagnosis were more likely to have involvement of extrathoracic lymph nodes whereas patients $40 \mathrm{yrs}$ of age and over were more likely to have abnormal calcium metabolism. He observed that nearly 50\% of his patients have intrathoracic involvement while the other half have one or more extrathoracic organ involvement. He reported that the skin was the mostly involved extrathoracic organ $(15.9 \%)$, followed by lymph node, eye, liver and erythema nodosum $(15.2,11.8,11.5,8.3 \%$ respectively) (3). Jerome and Johnson reported that $92 \%$ of their patients had intrathoracic sarcoidosis while $8 \%$ had extrathoracic involvement. The most frequent extrapulmonary involvement was skin $(20.6 \%$ in females and $16.9 \%$ in males). It was followed by peripheral lymphadenopathy, heart, liver, brain and eye in order. Routine chest roentgenographic findings $(46.5 \%)$, respiratory symptoms $(29.1 \%)$ and ocular symptoms (10.5\%) were substantially more frequent than any others (23). In our study $62 \%$ of the patients (38\% female, $24 \%$ male) had intrathoracic sarcoidosis while $38 \%$ (26\% female, $12 \%$ male) had extrathoracic involvements. Sixty of the patients (91\%) (65\% female, $35 \%$ male) with extrapulmonary involvement had also intrathoracic involvement. The most frequent involvement was the skin (20.6\% in females and $16.9 \%$ in males). There was no significant difference between the males and females as extrapulmonary involvement is considered.

As mentioned above sarcoidosis has a variable prognosis and it has been suggested to change according to ethnicity, age and gender. In some studies the rates of mortality 
were found to be higher among blacks than among whites and among women than among men in both blacks and whites $(10,15,32,33)$. Despite the idea that the progression of the disease changes according to geographic localisation and the belief that the disease in Europe has a better prognosis than the North America, it was shown that there was no significant difference in the prognosis in 1609 patients recruited from London, New York, Paris, Los Angeles and Tokyo in a recent multicentre retrospective study (3).

Jerome and Johnson reported that 12 out of 86 patients in their study group showed pulmonary fibrosis and 4 of them were progressive while the rest were nonprogressive; none of the patients died during the follow-up period (average 104 months) (6). Meanwhile 6 of 25 patients with the same characteristics died in a follow up period of 5 years (35). The different prognosis of these two groups may depend on their settlement (referral versus nonreferral). Unfavourable courses are referred to referral centers. It is clearly seen that the case weighted mortality rate is very low in $\mathrm{nRS}$ compared to RS (0.5 versus $4.8 \%)(15,24,31,34$, 35,36). (Table 6 and Table 7). Jerome suggested that the population served by referral settings RS are a composite of unselected patients derived from their serviced community plus a variable proportion of patients who have been referred, most often because of the expectation or realization of an unfavorable course.

In a Japanese study, most of the deaths were due to cardiac complications of sarcoidosis (77\%) $(11,2)$. In USA, $68 \%$ of the deaths were due to sarcoidosis and $87 \%$ of these deaths were secondary to pulmonary complications (pneumonia, pulmonary fibrosis and obstructive airway disease)
(17). In our study, $69 \%$ of the deaths were due to sarcoidosis and $64 \%$ of these deaths were the result of cardiac involvement while the rest $(36 \%)$ were due to pulmonary complications. It is seen that the percentage of death due to sarcoidosis is similar with the USA study (68\% versus $69 \%$ ), however, instead of pulmonary complications cardiac complications are the main cause of death. Despite the different percentage (69\% versus $77 \%$ ) cardiac death was the main cause of death like ours.

Comparing the age groups, mortality rates varied by race and sex. Among men and women of both races, the lowest age specific mortality rates were in people younger than 25 years.In Gideon's and Mannino's study the largest number of deaths $(28.3 \%)$ and the highest percentage of total deaths $(14 \%)$ occured in the 35 to 44 year age group (11). Among black men and women the highest mortality rates were in the 45 to 54 year age group, whereas among white men and women, the highest mortality rates were in the 75 to 84 -year age group (11). In our study we observed no death under 30 yrs and the 61-70 age group had the highest mortality rate for both genders. Fifty percent of total deaths occured in this group (37.5\% male and $62.5 \%$ female). It is similar with Gideon's study (11).

The authors found that the male to female mortality rates varied by age groups ( 0.55 in the 55-64 year old age group and 1.21 in the 15-24 year old age group) (11). We observed that the ratio was 2 in the 31-40 year old age group and 0.6 in the $61-70$ year old age group. The rates in the age groups of sixties are very similar and despite the different values in the youngs it is seen that the number of deaths of males are more than the females.

Tab. 6: The stages and the mortalities in referral settings (RS).

\begin{tabular}{|l|c|c|c|c|}
\hline Authors & number of cases & Stage I (\%) & Stage III-IV (\%) & Mortality (\%) \\
\hline Smellie,Hoyle & & 12 & 7.2 \\
\hline Neville,Walker & 125 & 38 & 13 & 6.1 \\
\hline Siltzbach et al $^{50}$ & 700 & 65 & 15 & 4.0 \\
\hline Scadding $^{27}$ & 1327 & 40 & 47 & 6.6 \\
\hline Sones,Israel $^{29}$ & 136 & 24 & 28 & 7.6 \\
\hline Chappel et al $^{31}$ & 199 & 36 & 22 & 4.2 \\
\hline Johnston $^{32}$ & 192 & 56 & 17 & 0 \\
\hline Case weighted mean (\%) & 159 & 51 & 17 & 4.8 \\
\hline Present study & & 49 & 11 & 11.6 \\
\hline
\end{tabular}

${ }^{++}$Calculated by Jerome MR (1) in his meta-analysis

Tab. 7: The stages and the mortalities in non-referral settings (nRS).

\begin{tabular}{|l|c|c|c|c|}
\hline Authors & number of cases & Stage I (\%) & Stage III-IV (\%) & Mortality (\%) \\
\hline Hillerdal,Nou $^{14}$ & 490 & 61 & 11 & 0.8 \\
\hline Reich,Johnson $^{33}$ & 79 & 53 & 24 & 0 \\
\hline Romer $^{8}$ & 243 & 56 & 5 & 0 \\
\hline Case-weighted mean (\%) $^{++}$ & & 59 & 11 & 0.5 \\
\hline
\end{tabular}

${ }^{++}$See in Tab. 6 
Jerome and Johnson reported that two cases died from hematologic disorders (acute myeloblastic leukemia and agnogenic myeloid metaplasia of the spleen) which can be interpreted as comorbidity (23). Compared with our 5 cases of comorbidity there is no similarity.

Stage was selected as the sole criterion of disease severity for comparison between studies because of its value as a prognostic guide, stage I being the most favorable and IV being the least one. Patients with stage IV have the highest mortality, and this stage correlates with most other prognostic signs such as chronicity, extrathoracic involvement, and progression $(2,23,36)$. In population based studies more than half of the patients were identified by mass population screening. The proportion of patients in each setting (RS and nRS) identified by mass population screening chest radiographs is an indicator of non-adverse selection; asymptomatic persons identified by this means would be expected to have the most favorable prognosis. The proportion identified by radiographic screening was higher in the nRS than in the RS compendia (24). The proportion of patients with stage I is directly related to the proportion identified by means of mass population radiographic screening. Huhti et al (18) had the highest percentage (64\%) reflecting the performance of triennial mass screening, followed by the series by Hillerdal et al (57\%)(15) and Romer (50\%) (34). In RS the proportions though lower than in nRS were substantial 34\% Siltzbach et al(36), 46\% Smellie and Hoyle (37), and 30\% Johnston (26) (Table 6 and Table 7).

X-ray graphics of the patients who were invited to our department were taken and compared with the initial ones. It was seen that the initial radiographic scannings showed that stage I was the most frequent one in both males and females. As we interpreted the current radiographic scanning we saw that stage 0 was most frequent in both gender.

At the time of diagnosis, the proportion of the first stage in our study is similar with the case weighted means of other RSs ( 50 versus 49 ), but the proportion of the third and fourth stage is lower than the case-weighted mean of RS (11 versus 17). It is similar with the proportions of the stage III-IV of nRS (11 versus 11) and had a lower proportion than the stage I of the nRS (50 versus 59). The proportion of the patients with the stage II was higher than the patients in both RS and nRS (39 versus 34 and 30). All of these may show that our center acts as the other RSs in mass population screening, but unfavourable cases are not referred to our center as much as to other RSs.

Jerome and Johnson (23) in a nonreferral setting, analysed the course and prognosis of 86 patients with sarcoidosis over a 10 year period of observation. Fifty three percent of the patients with intrathoracic sarcoidosis were found to have stage I disease at initial presentation and only $2.5 \%$ were found to have stage IV of the disease. Seventy six percent of the stage I cases resolved, $11 \%$ remained the same and $13 \%$ progressed to stage II. Sixty one percent of the stage II cases resolved, 22\% remained the same and $17 \%$ progressed to stage IV. Fifty six percent of the stage III cases resolved and $44 \%$ progressed to stage IV. Sixty six percent of the stage IV with pulmonary fibrosis remained the same while $34 \%$ showed progressive pulmonary fibrosis (23). In our study, $54 \%$ of stage I resolved and $46 \%$ progressed to stage II, $52 \%$ of stage II resolved, $28 \%$ remained the same and $20 \%$ progressed to stage III. Fifty five \% of stage III resolved and $45 \%$ progressed to stage IV.

As it is noticed, despite the many similarities we report, we have different values in mortality,survival rate and radiological staging, but it is emphasized by American Thoracic Society that it is difficult to evaluate the patients and define the life expectancy. They declared that as sarcoidosis is usually asymptomatic, its incidence and prevalance are markedly influenced by case ascertainment methods $(2,8)$. As among the races, sarcoidosis prevalence varies also by geographic region, both between countries and within countries $(6,16,39,40)$. In USA, the prevalance of sarcoidosis was higher in the Middle Atlantic and South Atlantic states for both whites and blacks and in rural areas compared with urban areas. Moriyama's study revealed the highest mortality to be in the South Atlantic states (29). Gideon and Mannino determined the lowest rate of mortality to be in Hawai. The reasons for this wide range are likely related to a difference in case ascertainment among different countries, in addition to any actual differences that exist (11).

We may have obtained different results due to these reported ethnic and geographic conditions. It is surprising that the prognosis in males is worse than in females in our center. It is a reality that the men in Turkey are under difficult social and economic conditions and they cannot take care of themselves as needed and they cannot take periodic controls. Therefore they are not diagnosed by radiologic scanning and referred to our RS as unfavourable cases. As a result, mortality may increase in males in Turkey.

\section{References}

1. ACCESS Research Group. Design of a case controlled etiologic study of sarcoidosis. (ACCES). J Clin Epidemiol 1999:52:1173-86.

2. American Thoracic Society, European Respiratory Society, World Association of Sarcoidosis and Other Granulamatous Diseases. Statement on sarcoidosis. Am J Respir Crit Care Med 1999;160:736-55.

3. Baughman RP, Teirstein AT, Judson MA et al. Clinical charecteristics of patients in a case control study of sarcoidosis (ACCESS). Am J Respir Crit Care Med 2001; 164:1885-9.

4. Bertin M, Fogdell HA, Olerup O et al. HLA-DR predicts the prognosis in Scandinavian patients with pulmonary sarcoidosis. AM J Respir Crit Care Med 1997:156:1601-5

5. Brennan NJ, Crean P, Long JP, Fitzgerald MX. High prevalance of familial sarcoidosis in an Irish population. Thorax 1984;39:14-8.

6. Brestniz EA, Strom BL. Epidemiology of sarcoidosis. Epidemiol Rev 1983; 5:124-56.

7. Chappel Ag, Cheung W, Hutchings HA. Sarcoidosis: a long term follow up study. Sarcoidosi Vasc Diffuse Lung Dis 2000;17:167-73.

8. Crystal RG. Sarcoidosis. In: Fauci AS, Braunwald E, Isselbacher KJ, eds. Harrison's principles of internal medicine. 14th ed. New York, NY: McGraw Hill, 1998: 1922-8.

9. Djuric B. Sarcoidosis in Eastern Europe. Sarcoidosis 1985;2:35-7.

10. Fraser RS, Müler NL, Colman N, Pare PD. Sarcoidosis. WB Saunders Comp Diagnosis of diseases of the chest. Fourth Ed. Philadelphia. 1999:1533-83.

11. Gideon NM, Mannino DM. Sarcoidosisi mortality in the United States, 
1979-1991: An analysis of multiple cause mortality data. Am J Med 1996; 100:423-7.

12. Gupta SK, Gupta S. Sarcoidosis in India: a review of 125 biopsy proven cases from eastern India. Sarcoidosis 1990;7:43-9.

13. Hannuksela M, Salo OP, Mustakallio KK. The prognosis of acute untreated sarcoidosis. Ann Klin Res 1970;2:57-64.

14. Henke CV, Henke G, Elvebeck LR et al. The epidemiology of sarcoidosis in Rochester, Minnesota: a population based study of incidence and survival. Am J Epidemiol 1986; 123:840-5.

15. Hillerdal G,Nou E, Osterman K, Schmekel B. Sarcoidosis: epidemiology and prognosis. A 15 year European study. Am Rev Respir Dis 1984:130:29-32.

16. Hosada Y, Hiraga Y, Odaca M et al. A cooperative study of sarcoidosis in Asia and Africa: analytic epidemiology. Ann NY Acad Sci 1976;278:355-67.

17. Huang Ct, Heurich AE, Sumon AL, Lyons HA. Mortality in sarcoidosis A changing pattern of the causes of death. Eur J Respir Dis 1981;62:231-8.

18. Huhti E,Poukkula A, Lilja M. Prognosis for sarcoidosis in a defined geographical area. Br J Dis Chest 1987;81:381-90.

19. Iwai K, Tachibana T, Takemura $T$ et al. Pathological studies on sarcoidosis autopsy. I. Epidemiological features of 320 cases in Japan. Acta Pathol Japan 1993;43:372-6.

20. Izumi T. Symposium:Population differences in clinical futures and progression of sarcoidosis throughout the world. Sarcoidosis 1992;9:105-18.

21. Jamaguchi M, Hosoda Y, Sasaki R, Aoki K. Epidemiological study on sarcoidosis in Japan. Recent trends in incidence and prevalance rates and changes in epidemiological features. Sarcoidosis 1989;61:138-46.

22. James DG. The many faces of sarcoidosis. The Thome Villar memorial lecture Sarcoidosis 1990;7:1-8

23. Jerome MR; Johnson RE. Course and prognosis of sarcoidosis in a nonreferra setting. Analysis of 86 patients observed for 10 years. Am J Med 1985;78:61-7.

24. Jerome MR. Mortality of intrathoracic sarcoidosis in Referral versus PopulationBased settings. Influence of stage, ethnicity and corticosteroid therapy. CHEST 2002; 121:32-5.

25. Jindal SK, Gupta D, Aggarwal AN. Sarcoidosis in developing countries. Curr Opin Pulm Med 2000; 6(5):448-54.

26. Johnston RN. Pulmonary sarcoidosis after ten to twenty years. Scott Med J 1986;31:72-8.

27. Lenner R,Schilero GJ, Padilla ML, Teirstein AS. Sarcoidosis presenting in patients older than 50 years.Sarcoidosis Vasc Diffuse Lung Dis 2002;19(2):143-7.

28. Martinetti M, Tinelli C, Kolek V et al. The sarcoidosis map; ajoint survey of clinical and immunogenetic findings in two European countries. Am J Respir Crit Care 1995;52:557-64.
29. Moriyama IM. Mortality from sarcoidosis in the United Studies. Am Review Respir Dis 1961;84(Suppl):116-9.

30. Neville E; Walker AN, Geraint James D. Prognostic factors predicting the outcome of sarcoidosis: an analysis of 818 patients. Q J Med 1983;208:525-33.

31. Reich JM, Johnson RE. Course and prognosis of sarcoidosis in a nonreferral setting: analysis of 86 patients observed for years. Am J Med 1985;78:61-7.

32. Reich JM, Johnson RE. Estimated incidence of clinically identified sarcoidosis in a Northwest United States population. Sarcoidosis. Vask Diffuse Lung Dis 1996;13:173-7

33. Reisner D.Observations on the course and prognosis of sarcoidosis. Am Rev Respir Dis 1967; 96:361-8.

34. Romer FK. Presentation of sarcoidosis and outcome of pulmonary changes. Dan Med Bul 1982; 29:27-32.

35. Scadding JG. Prognosis of intrathoracic sarcoidosis in England: a review of 136 cases after 5 years of observation. BMJ 1961; 4:1165-72.

36. Siltzbach LE, Geraint JDG, Neville E, et al. Course and prognosis of sarcoidosis around the world. Am J Med 1974;57:847-52.

37. Smellie H,Hoyle C. The natural history of pulmonary sarcoidosis. Q J Med 1960 29:539-58.

38. Sones M, Israel HL. Course and prognosis of sarcoidosis. Am J Med 1960;29: 84-93.

39. Teirstein AS, Lesser M. Worldwide distribution and epidemiology of sarcoidosis IN: James DG, editor. Sarcoidosis and other granulamatous diseases of the lung.New York: Marcel Decker, 1983. 101-34.

40. Young RC,Hackney R, Harden KA. Epidemiology of sarcoidosis: ethnic and geographic considerations. JAMA 1974;66:386-8.

Submitted June 2005.

Accepted March 2006.

Prof. Dr. Halil Yanardag, Istanbul Üniversitesi, Cerrahpaşa Tip Fakültesi, Aksaray, Istanbul, Turkey. e-mail: halilyanardag@yahoo.com 